Portland State University

PDXScholar

\title{
Discharge suppression system for a double focusing, atmospheric pressure ionization mass spectrometer
}

\author{
Andrew H. Grange \\ Robert J. O'Brien \\ Portland State University \\ Douglas F. Barofsky
}

Follow this and additional works at: https://pdxscholar.library.pdx.edu/chem_fac

Part of the Other Physics Commons

Let us know how access to this document benefits you.

\section{Citation Details}

Grange, A. H., O’Brien, R. J., \& Barofsky, D. F. (1988). Discharge suppression system for a double focusing, atmospheric pressure ionization mass spectrometer. Review Of Scientific Instruments, 59(4), 656.

This Article is brought to you for free and open access. It has been accepted for inclusion in Chemistry Faculty Publications and Presentations by an authorized administrator of PDXScholar. Please contact us if we can make this document more accessible: pdxscholar@pdx.edu. 
however, sufficient amplitude for chopping the beam can be produced only at the first resonance mode.

Although the modulation frequency is limited only to the first resonance frequency with a narrow bandwidth defined by its mechanical property, the chopper is operated remotely without electric connecting wires and is free from lubricating oil which is used in a conventional chopper. Therefore, the chopper proposed can be especially useful for chopping an optical beam or a neutral molecular beam in the experiments carried out in a vacuum chamber and in an electrically shielded vessel.

${ }^{1}$ A. C. Tam, Rev, Mod. Phys. 58, 381 (1986).

${ }^{2}$ P. Charpentier, F. Lepoutre, and L. Bertrand, J. Appl. Phys. 53, 608 (1982).

${ }^{3}$ G. Rousset, F. Lepoutre, and L. Bertrand, J. Appl. Phys. \$4, 2383 (1983).

\title{
Discharge suppression system for a double focusing, atmospheric pressure ionization mass spectrometer
}

\author{
Andrew H. Grange \\ Deparment of Environmental Science and Engineering, Oregon Graduate Center, 19600 N. W. Von \\ Neumann Drive, Beaverton, Oregon 97006 \\ Robert J. O'Brien \\ Department of Chemistry and Environmental Science Doctoral Program, Portland State University, \\ Portland, Orgeon 97207 \\ Douglas F. Barofsky \\ Department of Agriculturai Chemistry, Oregon State University, Corvallis, Oregon 97331 \\ (Received 30 November 1987; accepted for publication 5 January 1988)
}

An electrical discharge suppression system for a medium throughput $(\sim 2 \mathrm{l} / \mathrm{s})$ pumping line has been devised that works up to potentials of $\pm 15 \mathrm{kV}$. This device permits atmospheric pressure ionization sources to be interfaced to high-resolution, magnetic sector mass spectrometers with source potentials of $6-10 \mathrm{kV}$.

Atmospheric pressure ionization (API) mass spectrometry is used to study trace constituents in air without sample concentration or separation prior to analysis." Before we constructed our source, API mass spectrometry had generally been limited to quadrupole instruments that, at best, provide only unit resolution. ${ }^{1-3}$ High-performance, magnetic sector mass spectroneters operate with source potentials in the kilovolt range where arcing between an API source and grounded surfaces along connecting gas lines can disrupt operation, damage electronic components, and expose human operators to the hazard of a high-voltage shock.

The specific problems associated with electrical discharges through gas lines connected to a chemical ionization (Cl) source in a high-resolution mass spectrometer are known; they relate in all but one respect to the circumstances associated with using an API source in such an instrument, the exception being that the throughput of gas in the main pumping line of an API source is about 1000 times greater than that in the sample introduction line of a $\mathrm{Cl}$ source. We have devised an electrical discharge suppression system for a medium throughput $(\sim 21 / \mathrm{s})$ pumping line that overcomes this problem. Our discharge suppression system has permitted us to operate a VG $7070 \mathrm{E}-\mathrm{HF}$ mass spec- trometer in an API mode at a resolution of 4800 (10\% valley) at $\mathrm{m} / z$ 925 ; this capability has, for example, already allowed us to assign molecular formulas to at least two, previously unresolvable photo-oxidation products of toluene generated in an irradiated smog chamber. ${ }^{6}$

Figure 1 is a schematic diagram of a corona discharge API source. The corona region, where ions are formed, is normally at atmospheric pressure, and the differentially pumped region, the so-called collisionally induced dissociation (CID) region, separating the corona region from the mass analyzer is maintained at $\sim 1$ Torr. Therefore, during operation, the pressure within the pumping line falls from $\sim 1$ Torr in the CID region to $\sim 0.05$ Torr at the head of the pump. As in the case of $\mathrm{Cl}$, the conditions along such a gas line are ideal for glow discharges, particularly in the pressure range $0.1-0.3$ Torr. $^{4}$

The conditions under which gases in the pressure range of $10^{-2}$ to 1 Torr conduct electricity have been characterized. ${ }^{7-9}$ Such discharges are avalanche phenomena; their initiation occurs in accordance with Paschen's law, which states that the breakdown potential $V_{B}$ of a gas between two electrodes is a function of the product of the gas pressure and the electrode separation. ${ }^{5}$ Accordingly, for a given pressure 


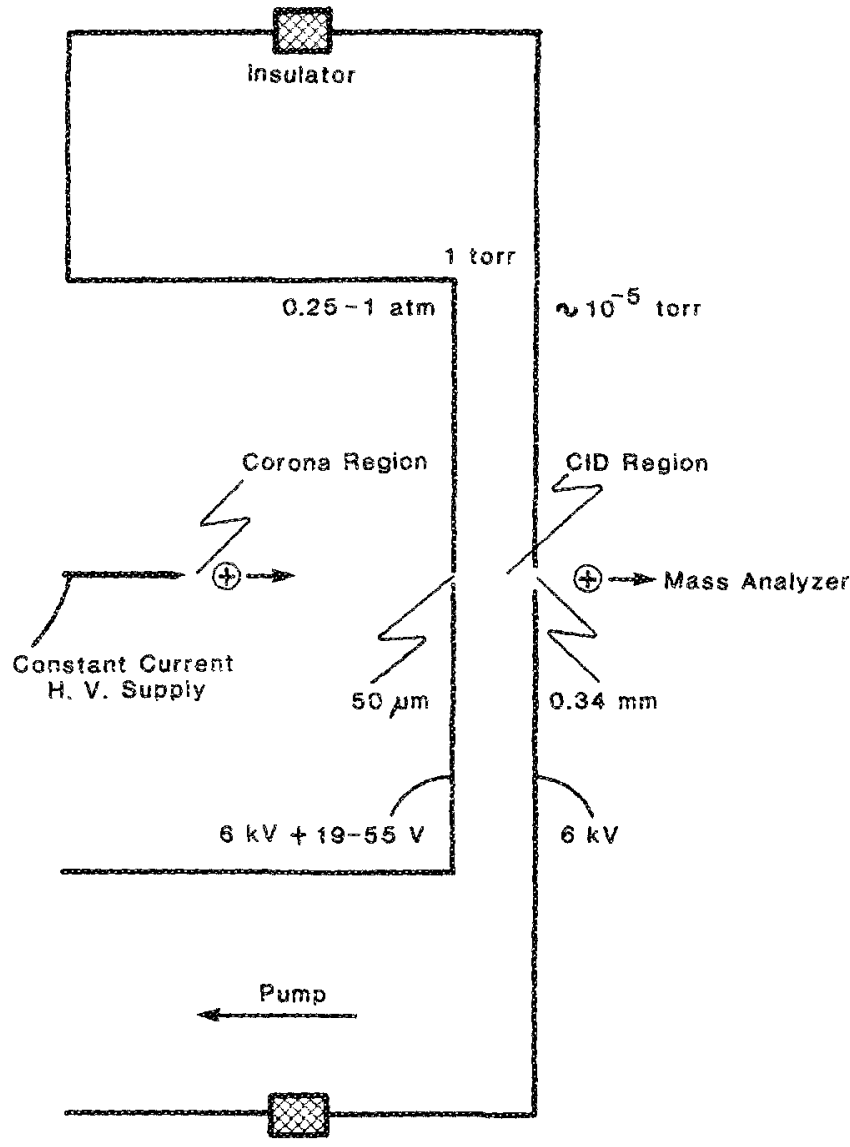

FIG. 1. Simplified drawing of a corona discharge API source showing the locations of the corona and collisionally induced dissociation (CDD) regions and of the apertures between those regions and the mass analyzer. Typical pressures within each region and typical potentials applied to the aperture electrodes are also indicated.

and voltage $F$, glow discharges can be suppressed by maintaining enough separation between the conducting elements in the gas volume to ensure that $V_{B}>V$. In practice, diserete insulating or resistive elements have been used to spread the drop in voitage over a series of stages that alternate with field-free regions where charge neutralization can occur. ${ }^{4,9}$

Initially, we based the design of our discharge suppression system soleiy on the proven concept of controlling the voltage gradient. However, with a voltage gradient device alone we still observed sporadic arcing at $6 \mathrm{kV}$ when the pressure in any region of the assembly was near 0.1 Torr. To eliminate this arcing, we altered our design: (1) to increase the gas pressure in the voltage gradient region and, thereby, in accordance with Paschen's law, raise $F_{B}$ and (2) to localize the pressure gradient in a region where the voltage is at ground potential and there is no electric field to initiate and sustain a discharge. A schematic of our fnal design is shown in Fig. 2 along with a graphic depiction of the system's operating principle.

Physically, the system consists of 16 ft of 3 -in.-i.d. PVC tubing, which is folded back on itself by means of a $U$ bend in the middle, with a total of 94 circular, stainless-steel screens ( 60 mesh $73 \%$ transparent) uniformly distributed ( $1 \frac{1}{2}$ in. apart) along the two, straight sections of pipe. The screendisks are electrically separated by a resistance of $1 \mathrm{M} \Omega$ that

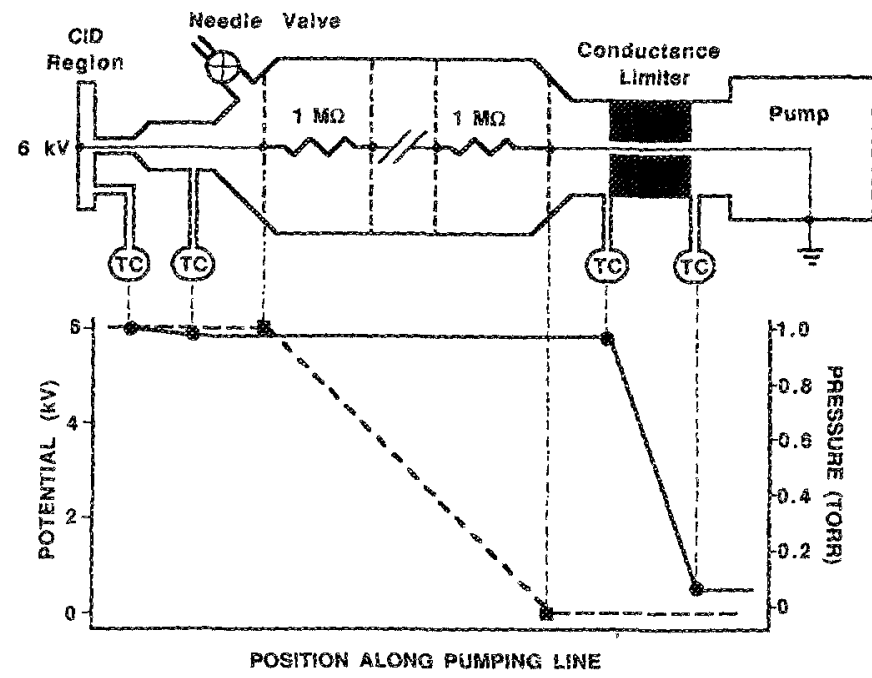

FrG. 2. Schematic diagram of the discharge suppression system. The plots illustrate how the system separates the potential (dashed) and pressure (solid) gradients along the pumping line to achieve discharge suppression.

actually consists of three, parallel $3-\mathrm{M} \Omega$ resistors spaced evenly around the circumference of a $1 \frac{5}{8}$-in.-diam circle $\left(\frac{11}{16}\right.$ in. from the inner surface of the PVC tubing); three resistors are used instead of one to give the assembly mechanical rigidity. The resistor-screen stages force the voltage gradient along the length of the pumping line to be uniform $(\sim 64 \mathrm{~V}$ per $1 \frac{1}{2}$ in. at $6 \mathrm{kV}$ ). The pressure along the voltage gradient region is maintained nearly constant at 1 Torr by bleeding air through a needle valve located in the pumping line at a point between where it exits the mass spectrometer and where the resistor-screen assembly begins and by placing a conductance limiting tube, 0.2-in. i.d. and 1.5-in. long, in the line just before it reaches the rotary pump. The pressure gradient is primarily confined to the flow limiter. The large diameter pipe and the highly transparent screens in the voltage gradient region constitute a high conductance line; hence, the conductance of the entire system is essentially equal to that of the flow limiter, namely $\sim 2 \mathrm{l} / \mathrm{s}$. ${ }^{10}$

Using an Al plug in place of the connection to the API source and a gas pressure of 1 Torr at the plug end, we tested our discharge suppression system up to $\pm 15 \mathrm{kV}$. With the plug at either $+15 \mathrm{kV}$ or $-15 \mathrm{kV}$; sporadic arcing was observed for periods of up to $45 \mathrm{~s}$ after turning on the highvoltage supply, but thereafter, no electrical breakdown was detected. Therefore, our system could be employed with double focusing mass spectrometers using accelerating voltages at least as high as $10 \mathrm{kV}$.

We believe that our present, prototype discharge suppression system is built very conservatively and that a more compact system containing fewer stages in a shorter, smaller diameter pipe would be just as effective. Such a device might allow higher accelerating voltages to be used with other types of ion sources currently limited by arcing at pressures greater than 0.1 Torr. ${ }^{11}$

This work was supported by the Environmental Protection Agency on Grant No. R811876. 
${ }^{1}$ N. M. Reid, J. B. French, J. A. Buckley, D. A. Lane, A. M. Lovert, and C. Rosenblat, in Proceedings of the 4th Joint Conference on Sensing Environmental Pollutants (ACS, Washington, DC, 1978), pp. 594-600.

${ }^{2}$ D. A. Lane, B. A. Thomson, A. M. Lovett, and N. M. Reid, in Advances in Mass Spectrometry, edited by A. Quayle (Heyder, London, 1980), Vol. 8B, pp. $1480-1489$.

${ }^{3}$ A. M. Lovett, N. M. Reid, J. A. Buckley, J. B. French, and D. M. Cameron, Biomed. Mass Spectrom. 6, 91 (1979).

${ }^{4}$ J. H. Futrell and L. H. Woicik, Rev. Sci. Instrum. 42, 244 (1971).

${ }^{5}$ A. H. Grange, R. J. O'Brien, and D. F. Barofsky, Rev. Sci. Instrum. 59. $573(1988)$.
${ }^{6}$ A. H. Grange, R. J. O'Brien, and D. F. Barofsky (submitted to Rapid Commun. Mass Spectrom.).

${ }^{7}$ F. M. Penning, Electrical Discharges in Gases (Macmillan, New York, 1957).

${ }^{8}$ C. L. Hemenway, R. W. Henry, and M. Caulton, Physical Electronics (Wiley, New York, 1962), Chap. 9, pp. 168-190.

'D. Thomas and W. L. Talbert, USAEC Report IS-1012, Ames, IA, 1964.

${ }^{10}$ S. Dushman, Scientific Foundations of Vacuum Techrique, 2nd ed., edited

by J. M. Laficrty (Wiley, New York, 1962), Chap. 2, pp. 80-117.

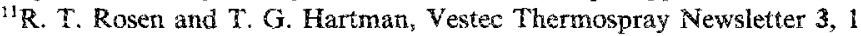
(1987).

\title{
Small pump for liquid nitrogen
}

\author{
Takeo Ebisuand Tadashi Watanabe \\ Deparment of Physics, Kobe University, Nada-ku, Kobe 657, Japan \\ (Received 6 October 1987; accepted for pubication 5 January 1988) \\ We present a small pump which is particularly useful when the conventional transfer method for \\ pressurizing liquid nitrogen is unavailable. Its transfer rate ranges from 0.2 to $0.81 / \mathrm{min}$ \\ depending on the speed of reciprocating motion of a piston driven by an electric motor.
}

Liquid nitrogen ( $\mathrm{LN}_{2}$ ) is not only a popular cryogen but is also indispensable for experiments at liquid-helium ( $\mathrm{LHe}$ ) temperature. After data taking has been finished or when any trouble has been noticed in the course of cooling a cryostat, the Dewar must be warmed back to room temperature as quickly as possible to save time. In order to help LHe evaporate, extracting $\mathrm{LN}_{2}$ from the Dewar in a mechanical way is preferable to its spontaneous evaporation alone. Otherwise, it will take a considerable period of time because of a large latent heat of vaporization of $\mathrm{LN}_{2}$. One of the most popular techniques for such a transfer is to pressurize $\mathrm{LN}_{2}$ to flow out through a delivery tube. However, we are oceasionally confronted with wide-mouthed Dewars, for which that procedure is really impossible. For a relatively small and light Dewar, it may be effective to pour out the $\mathrm{LN}_{2}$ by inclining the Dewar, but this is cumbersome and sometimes dangerous.

Hence, D. Chopra and H. Babb presented an alternative way by use of a pump system. ${ }^{1}$ The system is equipped with a motor at the bottom, so that the motor itself should be submerged in $\mathrm{LN}_{2}$. Their pump was designed to be $3.81 \mathrm{~cm}$ in width, which was mainly determined from the diameter of the motor. Unfortunately, we often find our $\mathrm{LN}_{2}$ Dewars too small to use their pump; in fact, an opening between two Dewars for $\mathrm{LN}_{2}$ and LHe is usually only $1.0-3.0 \mathrm{~cm}$ wide. A way out of such inconvenience is to make the motor smaller, or to separate it from the pump unit.

In this note, we present a pump system which is more compact and feasible than that in Ref. 1 . The pump system consists of a pump and a motor that are separable. Only the pump unit is shown in Fig. 1. The motor unit is omitted here because it is based on a conventional mechanism. A $0.3-\mathrm{mm}$ thick stainless-steel delivery tube of diameter $12.0 \mathrm{~mm}$ contains a brass piston which has a diameter $11.3 \mathrm{~mm}$ and a length of $10 \mathrm{~mm}$. A hole of diameter $4.8 \mathrm{~mm}$ is axially drilled through the piston and its upper circumference is reamed. We mount a stainless-steel ball of diameter $6.4 \mathrm{~mm}$ over the hole for a one-way valve. Another stainless-steel ball of the same size is also mounted as a valve at the bottom of the delivery tube. The piston performs a reciprocating motion. A long shaft made of brass is connected to a point $16 \mathrm{~mm}$ away from the center of a flywheel of diameter $40 \mathrm{~mm}$ through a brass crankshaft of length $80 \mathrm{~mm}$. The piston stroke is $32 \mathrm{~mm}$. The flywheel is driven by an inexpensive dc motor via a gear box used in toys. Particular care is required

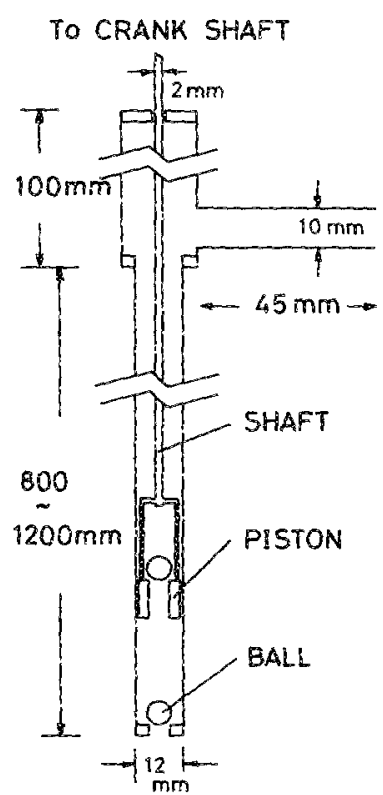

FIG. 1. Cross-sectional view of the pump unit. The motor unir is omitted for simplicity. 